\title{
ON RIGHT HEREDITARY SPSD-RINGS OF BOUNDED REPRESENTATION TYPE I
}

\author{
Nadiya Gubareni \\ Institute of Mathematics, Czestochowa University of Technology \\ Czestochowa, Poland \\ nadiya.gubareni@yahoo.com
}

\begin{abstract}
The structure of right hereditary semiperfect semidistributive rings of bounded representation type is described in terms of Dynkin diagrams and diagrams with weights. We describe it using a reduction to mixed matrix problems.
\end{abstract}

\section{Introduction}

This paper is devoted to the study of boundedness of right hereditary semiperfect semidistributive rings (SPSD-rings, in short) considered in [1] and is a continuation of it. These rings were first described in [2].

We use notation and definitions of articles $[1,3,4]$ and books $[5,6]$.

Recall that $\operatorname{ring} A$ has a bounded representation type if there is an upper bound on the number of generators required for indecomposable finitely presented $A$-modules. Otherwise it is of the unbounded representation type.

In this paper we prove the necessity of the following main theorem which gives the structure of right hereditary SPSD-rings of bounded representation type in terms of Dynkin diagrams and diagrams with weights:

Theorem 1. Let $\left\{O_{i}\right\}$ be a family of discrete valuation rings with a common skew field of fractions $D$, and let $\mathrm{S}=\mathrm{S}_{0} \cup \mathrm{S}_{1}$ be a disjoint union of subposets. A right hereditary SPSD-ring A is of bounded representation type if and only if $A=A(\mathrm{~S}, O)$ and the undirected graph $\overline{\mathbf{0}(\mathrm{S})}$ of the Hasse diagram $\mathbf{\square}(\mathrm{S})$ of the poset $\mathrm{S}$ is a finite disjoint union of Dynkin diagrams of the type $\mathrm{A}_{n}, \mathrm{D}_{n}, \mathrm{E}_{6}, \mathrm{E}_{7}, \mathrm{E}_{8}$ and the following diagrams with weights:

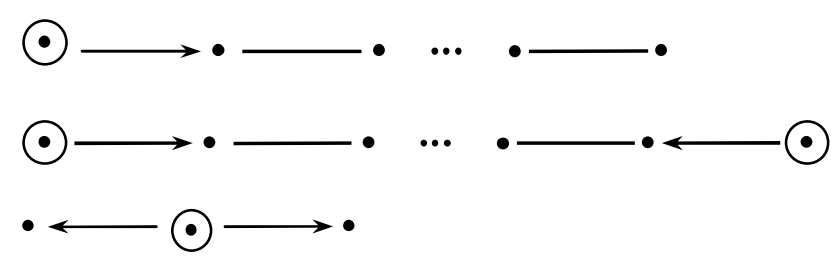




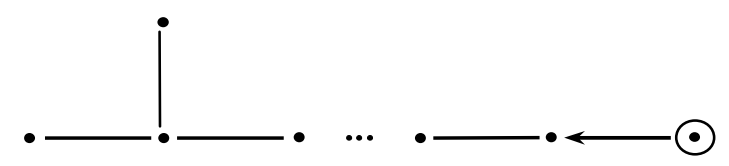

where all vertices with weight 1 correspond to the minimal elements of the poset $\mathrm{S}$.

Note that this theorem was first formulated in [7], where it is given without proof, and it can be considered as a simple generalization of [8, Theorem I]. In this paper we give two different proofs of the necessity of this theorem using the results of $[3,8,9]$.

All rings considered in this paper are assumed to be associative (but not necessary commutative) with $1 \neq 0$, and all modules are assumed to be unital.

\section{Preliminaries}

According Gabriel [10] and Dlab and Ringel [11], a hereditary finite dimensional algebra is of finite representation type if and only if the corresponding diagram is a Dynkin diagram of the type $\mathrm{A}_{n}, \mathrm{~B}_{n}, \mathrm{C}_{n}, \mathrm{D}_{n}, \mathrm{E}_{6}, \mathrm{E}_{7}, \mathrm{E}_{8}, \mathrm{~F}_{4}$ or $\mathrm{G}_{2}$. From this fundamental result [1, Theorem 3] and [1, Proposition 4] we immediately obtain the following statement:

Proposition 2. If diagram $\Gamma(\mathrm{S})$ of a poset $\mathrm{S}$ is not a disjoint union of the Dynkin diagrams of the the type $\mathrm{A}_{n}, \mathrm{D}_{n}, \mathrm{E}_{6}, \mathrm{E}_{7}, \mathrm{E}_{8}$, then the right hereditary SPSD-ring $A=A(\mathrm{~S}, O)$ is a ring of the unbounded representation type.

Proposition 3. If ring $A$ is of the bounded representation type then each of its minors is of bounded representation type too.

Proof. Let $P$ be a finitely generated projective $A$-module, $B=\operatorname{End}_{A}(P)$, and let $M$ be a finitely presented $B$-module. Then there are the exact sequences:

$$
\begin{aligned}
& 0 \rightarrow X \rightarrow B^{n} \rightarrow M \rightarrow 0 \\
& 0 \rightarrow Y \rightarrow B^{m} \rightarrow X \rightarrow 0
\end{aligned}
$$

Denote by $C(P)$ the full subcategory of the category of all $A$-modules consisting of $A$-modules $M$ such that there exists an exact sequence

$$
P^{J} \rightarrow P^{I} \rightarrow M \rightarrow 0
$$

where $P^{I}$ denotes a direct sum of modules isomorphic to $P$. By the Morita theorem [5, Theorem 10.7.2], it follows that the categories $B$-mod and $C(P)$ are equivalent, 
hence there is an $A$-module $M^{\prime} \in C(P)$ such that $M=F(M)=\operatorname{Hom}_{A}(P, M)$. Therefore there is a sequence

$$
0 \rightarrow X \rightarrow B^{n} \rightarrow F\left(M^{\prime}\right) \rightarrow 0
$$

Applying the exact functor $G=* \otimes_{B} P$ to the exact sequences (2) and (4), we get

$$
\begin{gathered}
0 \rightarrow G(X) \rightarrow P^{n} \rightarrow M^{\prime} \rightarrow 0 \\
0 \rightarrow G(Y) \rightarrow P^{m} \rightarrow G(X) \rightarrow 0
\end{gathered}
$$

Hence $\mu_{A}(M)=\mu_{A}\left(P^{n}\right)-\mu_{A}(G(X))=n s-\mu_{A}(G(X))$, where $s=\mu_{A}(P)$, and $\mu_{A}(U)$ is the minimum number of generators of an $A$-module $U$.

Since $A$ is a ring of the bounded representation type, there exists a number $N$ such that $\mu_{A}(U) \leq N$ for any $A$-module $U$. Therefore $\mu_{A}(M) \leq N$ and $\mu_{A}(G(X)) \leq N$, that is, $n s=\mu_{A}(M)+\mu_{A}(G(X)) \leq 2 N$, i.e. $n \leq 2 N / \mathrm{s}$. Writing $2 N / s=N_{1}$ we obtain that $\mu_{B}(M) \leq n \leq N_{1}$ and this is true for any finitely presented $B$-module $M$. Therefore $B$ is a ring of bounded representation type.

\section{Mixed matrix problems and posets}

Let $O$ be a discrete valuation ring with a skew field of fractions $D$ and the Jacobson radical $R=\pi O=O \pi$.

By left $O$-elementary transformations of rows of a matrix $\mathbf{X}$ we mean the transformations of the following three types:

(a) interchanging of two rows;

(b) multiplications of a row on the left by an invertible element of $O$;

(c) addition of a row multiplied on the left by an arbitrary element of $O$ to another row.

In a similar way we define left $D$-elementary transformations of rows and, by symmetry, right $O$-elementary and right $D$-elementary transformations of columns.

Let $\mathbf{T}=\left(\mathbf{T}_{i j}\right)$ be a rectangular matrix with entries in $D$ partitioned into $n$ horizontal strips $\left\{\mathbf{T}_{i}\right\}_{i=1, \ldots, n}$ and $m$ vertical strips $\left\{\mathbf{T}^{j}\right\}_{j=1, \ldots, m}$ so that a block $\mathbf{T}_{i j}$ is the intersection of the $i$-th horizontal strip $\mathbf{T}_{i}$ and the $j$-th vertical strip $\mathbf{T}^{j}$.

Let $\mathrm{M}_{n}(D)$ be the ring of $n \times n$ matrices over $D$ with matrix units $e_{i j}$. Following [8] we consider a transforming algebra $X=\bigoplus_{i, j=1}^{n} e_{i j} X_{i j} \subseteq \mathrm{M}_{n}(D)$ such that:

(a) $X_{i i}=O$ or $X_{i i}=D$;

(b) $X_{i j} X_{j k} \subseteq X_{i k}$

(c) $X_{i j} X_{j i} \neq X_{i i}$ for $i \neq j$.

for each $i, j, k=1,2, \ldots, n$.

Obviously, $X_{i j}=D$ or $X_{i j}=\pi^{\alpha_{i j}} O$, where $\alpha_{i j} \in \mathbf{Z}$. We set $D=\pi^{-\infty} O$ and $0=\pi^{+\infty} O$. 
A family of elementary transformations over the row strips of a rectangular matrix $\mathbf{T}=\left(\mathbf{T}_{i j}\right)$ of the following form:

(i) left $X_{i i}$-elementary transformations of rows in the strip $\mathbf{T}_{i}$;

(ii) addition of rows in a strip $\mathbf{T}_{i}$ multiplied on the left by elements of $X_{i j}$ to rows of a strip $\mathbf{T}_{j}$

will be called admissible transformations with respect to an algebra $X$.

In a similar way one can define admissible transformations over the column strips of a matrix $\mathbf{T}$ with respect to an algebra $Y=\bigoplus_{i, j=1}^{m} e_{i j} Y_{i j} \subseteq \mathrm{M}_{m}(D)$.

The dimension of a stripped matrix $\mathbf{T}$ is the vector

$$
\mathbf{d}=\mathrm{d}(\mathbf{T})=\left(d_{1}, d_{2}, \ldots, d_{n} ; d^{1}, d^{2}, \ldots, d^{m}\right),
$$

where $d_{i}$ is the number of rows of the $i$-th horizontal strip $\mathbf{T}_{i}$ and $d^{j}$ is the number of columns of the $j$-th vertical strip $\mathbf{T}^{j}$ for $j=1, \ldots, m$. We set

$$
\operatorname{dim}(\mathbf{T})=\sum_{i=1}^{n} d_{i}+\sum_{j=1}^{m} d^{j}
$$

According to [9], a mixed matrix problem has a bounded representation type, if there is a constant $C$ such that $\operatorname{dim}(\mathbf{T})<C$ for all indecomposable matrices $\mathbf{T}$.

\section{Flat mixed matrix problem:}

Given a triangular matrix $\mathbf{T}=\left(\mathbf{T}_{\mathrm{ij}}\right)$ with entries in D partitioned into $n$ horizontal strips $\left\{\mathbf{T}_{i}\right\}_{i=1, \ldots, n}$ and $m$ vertical strips $\left\{\mathbf{T}^{j}\right\}_{j=1, \ldots, m}$, two transforming algebras $X \subseteq \mathrm{M}_{n}(D)$ and $Y \subseteq \mathrm{M}_{m}(D)$. One performs admissible transformations over row strips with respect to $X$ and admissible transformations over column strips with respect to $Y$. Define the boundedness type of this matrix problem.

This matrix problem was solved in [9] in terms of critical pairs of sets in the sense of Kleiner [12].

Recall that a totally ordered set consisted of $n$ elements is called a chain and denoted by $(n)$. A cardinal sum of $k$ chains consisting of $n_{1}, n_{2}, \ldots, n_{k}$ elements is denoted by $\left(n_{1}, n_{2}, \ldots, n_{k}\right)$. A cardinal sum of posets $P$ and $Q$ is denoted by $P \leq Q$. Denote by $N$ the poset $\{a<b>c<d\}$.

Associate with a transforming algebra $X$ a poset $P(X)=\sum_{i=1}^{n} P_{i}$, which is a cardinal sum of posets $P_{i}$, where $P_{i}$ is a chain of the following type:

(a) $P_{i}=\left\{p_{i}^{0}\right\}$ is a one-point chain if $X_{i i}=D$;

(b) $P_{i}=\left\{p_{i}^{k}\right\}_{k \in \mathbf{Z}}$ is an infinite chain if $X_{i i}=O$.

The order relation in $P(X)$ is defined as follows:

$$
p_{i}^{k} \leq p_{i}^{l} \Leftrightarrow k-l \geq \alpha_{i j} \text { if } X_{i j}=\pi^{\alpha_{i j}} O .
$$




\section{Definition 1.}

A pair $(P, Q)$ of posets is called a critical pair of sets (in the sense of Kleiner) if one of the following conditions is satisfied up to the transposition of $P$ and $Q$ :

- $P=(1) ; \quad Q=(1,1,1,1) \vee(2,2,2) \vee(1,3,3) \vee(1,2,5) \vee N \leq 4$;

- $P=(2) ; \quad Q=(1,1,1) \vee(3,3) \vee(2,5)$;

- $P=(3) ; \quad Q=(2,2) \vee(1,5)$;

- $P=(4) ; \quad Q=(1,3)$;

- $P=(5) ; \quad Q=N$;

- $P=(6) ; \quad Q=(1,2)$;

- $P=(1,1) ; Q=(1,1)$.

Theorem 4 [9]. A flat matrix problem defined by a pair of transforming algebras $(\mathrm{X}, \mathrm{Y})$ of the above type is of bounded representation type if and only if the pair of partially ordered sets $(\mathrm{P}(\mathrm{X}), \mathrm{P}(\mathrm{Y}))$ contains no critical pairs of sets in the sense of Kleiner.

\section{Proof of the necessity in Theorem 1}

Lemma 5. Let $O$ be a discrete valuation ring with a skew field of fractions $D$ and the radical $R=\pi O=O \pi$. Then the ring

$$
A=\left(\begin{array}{llll}
O & D & D & D \\
0 & D & 0 & 0 \\
0 & 0 & D & 0 \\
0 & 0 & 0 & D
\end{array}\right),
$$

corresponding to the diagram

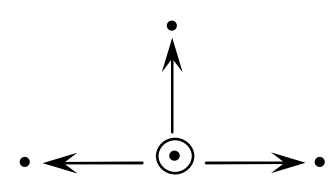

is a ring of unbounded representation type.

Proof. Let $M$ be a finitely generated right $A$-module that is given by the set $\left\{t ; l_{1}, l_{2}, l_{3} ; \mathbf{T}\right\}$, in which the matrix $\mathbf{T}$ has the following form:

\begin{tabular}{|c|c|c|c|}
\hline $\mathbf{E}$ & $\mathbf{T}_{12}$ & $\mathbf{T}_{13}$ & $\mathbf{T}_{14}$ \\
\hline $\mathbf{O}$ & $\mathbf{E}$ & $\mathbf{O}$ & $\mathbf{O}$ \\
\hline $\mathbf{O}$ & $\mathbf{O}$ & $\mathbf{E}$ & $\mathbf{O}$ \\
\hline $\mathbf{O}$ & $\mathbf{O}$ & $\mathbf{O}$ & $\mathbf{E}$ \\
\hline
\end{tabular}


where $\mathbf{T}_{1 i} \in \mathrm{M}_{t \times l_{i}}(D)(i=2,3,4)$ are matrices over $D$. The matrix of transformations $\mathbf{U}$ has the following form:

\begin{tabular}{|c|c|c|c|}
\hline $\mathbf{U}_{11}$ & $\mathbf{O}$ & $\mathbf{O}$ & $\mathbf{O}$ \\
\hline $\mathbf{O}$ & $\mathbf{U}_{22}$ & $\mathbf{O}$ & $\mathbf{O}$ \\
\hline $\mathbf{O}$ & $\mathbf{O}$ & $\mathbf{U}_{33}$ & $\mathbf{O}$ \\
\hline $\mathbf{O}$ & $\mathbf{O}$ & $\mathbf{O}$ & $\mathbf{U}_{44}$ \\
\hline
\end{tabular}

where $\mathbf{U}_{11}$ is an invertible matrix with entries in $O$, and $\mathbf{U}_{i i}(i=2,3,4)$ are invertible matrices with entries in $D$. Reducing the matrix $\mathbf{T}$ by the matrix $\mathbf{U}$ leads to the following matrix problem, given by a matrix $\mathbf{T}_{1}$

$$
\begin{array}{|l|l|l|}
\hline \mathbf{A}_{1} & \mathbf{A}_{2} & \mathbf{A}_{1} \\
\hline
\end{array}
$$

and the following admissible transformations:

(a) left $O$-elementary transformations of rows of the matrix $\mathbf{T}_{1}$;

(b) right $D$-elementary transformations of columns inside any vertical strip $\mathbf{A}_{i}$ $(i=1,2,3)$.

Set

$$
\mathbf{A}_{1}=\left(\begin{array}{cccc}
1 & 0 & \cdots & 0 \\
0 & 1 & \cdots & 0 \\
\vdots & \vdots & \ddots & \vdots \\
0 & 0 & \cdots & 0 \\
0 & 0 & \cdots & 0 \\
0 & 0 & \cdots & 0 \\
\vdots & \vdots & \ddots & \vdots \\
0 & 0 & \cdots & 0
\end{array}\right), \quad \mathbf{A}_{2}=\left(\begin{array}{cccc}
\pi^{-2} & 0 & \cdots & 0 \\
0 & \pi^{-4} & \cdots & 0 \\
\vdots & \vdots & \ddots & \vdots \\
0 & \underline{0} & \cdots & \frac{\pi^{-2 n}}{1} \\
0 & \cdots & 0 \\
0 & 1 & \cdots & 0 \\
\vdots & \vdots & \ddots & \vdots \\
0 & 0 & \cdots & 1
\end{array}\right), \quad \mathbf{A}_{3}=\left(\begin{array}{c}
\pi^{n-1} \\
\pi^{n-2} \\
\vdots \\
1 \\
0 \\
0 \\
\vdots \\
0
\end{array}\right)
$$

where $\pi \in R=\operatorname{rad} O, \pi \neq 0$. By [4, Lemma 3], the matrix $\mathbf{T}_{1}$ is indecomposable and therefore ring $A$ is of unbounded representation type.

\section{Remark 1.}

A mixed matrix problem over a matrix $T_{1}$ is defined by two transforming algebras:

$$
X=O, \quad Y=\left(\begin{array}{ccc}
D & 0 & 0 \\
0 & D & 0 \\
0 & 0 & D
\end{array}\right)
$$


Correspondingly, $P(X)$ is an infinite chain, and $P(Y)=\{1,1,1\}$. Therefore the pair of posets $\{P(X), P(Y)\}$ contains a critical pair of sets $\{(2),(1,1,1\}\}$. By theorem 4 this matrix problem is of unbounded representation type.

Lemma 6. Let $O$ be a discrete valuation ring with a skew field of fractions $D$ and the radical $R=\pi O=O \pi$. Then the ring

$$
\mathbf{A}=\left(\begin{array}{llll}
O & 0 & 0 & D \\
0 & O & 0 & D \\
0 & 0 & D & D \\
0 & 0 & 0 & D
\end{array}\right),
$$

corresponding to the diagram

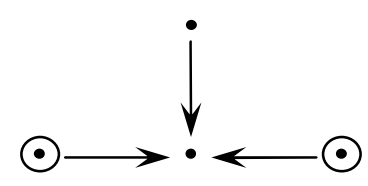

is a ring of the unbounded representation type.

Proof. Let $M$ be a finitely generated right $A$-module which is given by the set $\left\{t_{1}, t_{2} ; l_{1}, l_{2} ; \mathbf{T}\right\}$, in which the matrix $\mathbf{T}$ has the following form:

\begin{tabular}{|c|c|c|c|}
\hline $\mathbf{E}$ & $\mathbf{O}$ & $\mathbf{O}$ & $\mathbf{T}_{14}$ \\
\hline $\mathbf{O}$ & $\mathbf{E}$ & $\mathbf{O}$ & $\mathbf{T}_{24}$ \\
\hline $\mathbf{O}$ & $\mathbf{O}$ & $\mathbf{E}$ & $\mathbf{T}_{34}$ \\
\hline $\mathbf{O}$ & $\mathbf{O}$ & $\mathbf{O}$ & $\mathbf{E}$ \\
\hline
\end{tabular}

where $\mathbf{T}_{i 4} \in \mathrm{M}_{t_{i} \times l_{2}}(D)(i=1,2)$ and $\mathbf{T}_{34} \in \mathrm{M}_{l_{1} \times l_{2}}(D)$ are matrices over $D$. The matrix of transformations $\mathbf{U}$ has the following form:

\begin{tabular}{|c|c|c|c|}
\hline $\mathbf{U}_{11}$ & $\mathbf{O}$ & $\mathbf{O}$ & $\mathbf{O}$ \\
\hline $\mathbf{O}$ & $\mathbf{U}_{22}$ & $\mathbf{O}$ & $\mathbf{O}$ \\
\hline $\mathbf{O}$ & $\mathbf{O}$ & $\mathbf{U}_{33}$ & $\mathbf{O}$ \\
\hline $\mathbf{O}$ & $\mathbf{O}$ & $\mathbf{O}$ & $\mathbf{U}_{44}$ \\
\hline
\end{tabular}

where $\mathbf{U}_{i i}$ is an invertible matrix with entries in $O(i=1,2)$ and $\mathbf{U}_{i i}(i=3,4)$ are invertible matrices with entries in $D$. Reducing the matrix $\mathbf{T}$ by the matrix $\mathbf{U}$ is equivalent to the matrix problem given by a matrix $\mathbf{T}_{1}$ 


\section{\begin{tabular}{|l|l|l|}
\hline $\mathbf{A}_{1}$ & $\mathbf{A}_{2}$ & $\mathbf{A}_{3}$ \\
\hline
\end{tabular}}

and the following admissible transformations:

(a) left $D$-elementary transformations of rows of the matrix $\mathbf{T}_{1}$;

(b) right $O$-elementary transformations of columns inside any block $\mathbf{A}_{i}(i=1,2)$;

(c) right $D$-elementary transformations of columns inside the block $\mathbf{A}_{3}$.

Set

$$
\mathbf{A}_{1}=\left(\begin{array}{cccc}
1 & 0 & \cdots & 0 \\
0 & 1 & \cdots & 0 \\
\vdots & \vdots & \ddots & \vdots \\
0 & 0 & \cdots & 1
\end{array}\right), \quad \mathbf{A}_{2}=\left(\begin{array}{cccc}
\pi^{2} & 0 & \cdots & 0 \\
0 & \pi^{4} & \cdots & 0 \\
\vdots & \vdots & \ddots & \vdots \\
0 & 0 & \cdots & \pi^{2 n}
\end{array}\right), \quad \mathbf{A}_{3}=\left(\begin{array}{c}
1 \\
\pi \\
\vdots \\
\pi^{n-1}
\end{array}\right) .
$$

By [4, Lemma 3], the matrix $\mathbf{T}_{1}$ is indecomposable. So the corresponding module $M$ is indecomposable and the ring $A$ is of the unbounded representation type.

\section{Remark 2.}

A mixed matrix problem over a matrix $T_{1}$ is defined by transforming algebras:

$$
X=D, \quad Y=\left(\begin{array}{ccc}
O & 0 & 0 \\
0 & O & 0 \\
0 & 0 & D
\end{array}\right)
$$

Correspondingly, we have two posets: $P(X)=(1)$ is a one-point chain (1), and $P(Y)$ is a cardinal sum of two infinite chains and a one-point chain (1). Therefore the pair of posets $\{P(X), P(Y)\}$ contains a critical pair of sets $\{(1),(3,3,1\}\}$. By theorem 4 this matrix problem is of the unbounded representation type.

Lemma 7. Let $O$ be a discrete valuation ring with a skew field of fractions $D$ and the radical $R=\pi O=O \pi$. Then the ring

$$
\mathbf{A}=\left(\begin{array}{llll}
O & 0 & D & D \\
0 & O & D & D \\
0 & 0 & D & D \\
0 & 0 & 0 & D
\end{array}\right)
$$

corresponding to the diagram

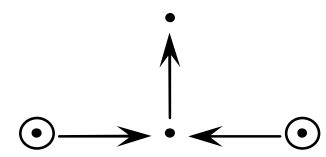

is a ring of the unbounded representation type. 
The proof of this lemma is the same as for Lemma 6 .

Lemma 8. Let $O$ be a discrete valuation ring with a skew field of fractions $D$ and the radical $R=\pi O=O \pi$. Then the ring

$$
\mathbf{A}=\left(\begin{array}{llll}
O & 0 & D & D \\
0 & D & 0 & D \\
0 & 0 & D & 0 \\
0 & 0 & 0 & D
\end{array}\right),
$$

corresponding to the diagram

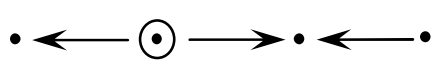

is a ring of the unbounded representation type.

Proof. Let $M$ be a finitely generated right $A$-module that is given by the set $\left\{t ; l_{1}, l_{2}, l_{3} ; \mathbf{T}\right\}$, in which the matrix $\mathbf{T}$ has the following form:

\begin{tabular}{|c|c|c|c|}
\hline $\mathbf{E}$ & $\mathbf{O}$ & $\mathbf{T}_{13}$ & $\mathbf{T}_{14}$ \\
\hline $\mathbf{O}$ & $\mathbf{E}$ & $\mathbf{O}$ & $\mathbf{T}_{24}$ \\
\hline $\mathbf{O}$ & $\mathbf{O}$ & $\mathbf{E}$ & $\mathbf{O}$ \\
\hline $\mathbf{O}$ & $\mathbf{O}$ & $\mathbf{O}$ & $\mathbf{E}$ \\
\hline
\end{tabular}

where $\mathbf{T}_{13} \in \mathrm{M}_{t \times l_{1}}(D), \mathbf{T}_{14} \in \mathrm{M}_{t \times l_{2}}(D)$, and $\mathbf{T}_{24} \in \mathrm{M}_{l_{3} \times l_{2}}(D)$. The matrix of transformations $\mathbf{U}$ has the following form:

\begin{tabular}{|c|c|c|c|}
\hline $\mathbf{U}_{11}$ & $\mathbf{O}$ & $\mathbf{O}$ & $\mathbf{O}$ \\
\hline $\mathbf{O}$ & $\mathbf{U}_{22}$ & $\mathbf{O}$ & $\mathbf{O}$ \\
\hline $\mathbf{O}$ & $\mathbf{O}$ & $\mathbf{U}_{33}$ & $\mathbf{O}$ \\
\hline $\mathbf{O}$ & $\mathbf{O}$ & $\mathbf{O}$ & $\mathbf{U}_{44}$ \\
\hline
\end{tabular}

where $\mathbf{U}_{11}$ is an invertible matrix with entries from $O$, and $\mathbf{U}_{i i}(i=2,3,4)$ are invertible matrices with entries from $D$. Reducing the matrix $\mathbf{T}$ by the matrix $\mathbf{U}$ is equivalent to the matrix problem given by a matrix $\mathbf{T}_{1}$

\begin{tabular}{|l|l|}
\hline $\mathbf{A}_{1}$ & $\mathbf{A}_{2}$ \\
\hline $\mathbf{O}$ & $\mathbf{A}_{3}$ \\
\hline
\end{tabular}


and the following admissible transformations:

(a) left $O$-elementary ( $D$-elementary) transformations of rows inside the first (second) horizontal strip of the matrix $\mathbf{T}_{1}$;

(b) right $D$-elementary transformations of columns inside each vertical strip of the matrix $\mathbf{T}_{1}$.

Reducing the matrix $\mathbf{A}_{3}$ to the form

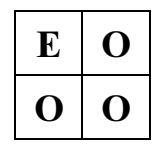

we get that the matrix $\mathbf{A}_{2}$ has the form:

$$
\begin{array}{|l|l|}
\hline \mathbf{B}_{1} & \mathbf{B}_{2} \\
\hline
\end{array}
$$

We can add any column of $\mathbf{B}_{2}$ multiplied on the right by elements of $D$ to any column of $\mathbf{B}_{1}$. Thus the matrices $\mathbf{A}_{1}, \mathbf{B}_{1}$ and $\mathbf{B}_{2}$ form the matrix problem considered in [4, Problem II] . By [4, Lemma 4] the ring $A$ is of the unbounded representation type.

\section{Remark 3.}

A mixed matrix problem which forms matrices $\mathbf{A}_{1}, \mathbf{B}_{1}$ and $\mathbf{B}_{2}$ is defined by transforming algebras:

$$
X=O, \quad Y=\left(\begin{array}{ccc}
D & D & 0 \\
0 & D & 0 \\
0 & 0 & D
\end{array}\right)
$$

Correspondingly, we have two posets: $P(X)$ is an infinite chain and $P(Y)$ is a cardinal sum $(1,2)$. Therefore the pair of posets $\{P(X), P(Y)\}$ contains a critical pair of sets $\{(6),(1,2)\}$. By Theorem 4 this matrix problem is of unbounded representation type.

Analogously, one can prove the following lemma:

Lemma 9. Let $O$ be a discrete valuation ring with a skew field of fractions $D$ and the radical $R=\pi O=O \pi$. Then the ring

$$
\mathbf{A}=\left(\begin{array}{llll}
O & D & D & D \\
0 & D & 0 & 0 \\
0 & 0 & D & D \\
0 & 0 & 0 & D
\end{array}\right),
$$


corresponding to the diagram

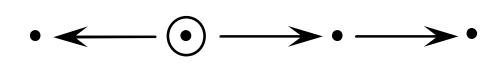

is a ring of the unbounded representation type.

Lemma 10. Let $O$ be a discrete valuation ring with a skew field of fractions $D$ and the Jacobson radical $R=\pi O=O \pi$. Then the ring

$$
\mathbf{A}=\left(\begin{array}{lllll}
O & 0 & 0 & 0 & D \\
0 & D & 0 & 0 & D \\
0 & 0 & D & D & D \\
0 & 0 & 0 & D & 0 \\
0 & 0 & 0 & 0 & D
\end{array}\right)
$$

corresponding to the diagram

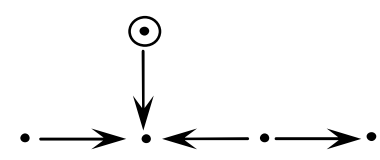

is a ring of the unbounded representation type.

Proof. Let $M$ be a finitely generated right $A$-module which is given by the set $\left\{t ; l_{1}, l_{2}, l_{3}, l_{4} ; \mathbf{T}\right\}$, in which the matrix $\mathbf{T}$ has the following form:

\begin{tabular}{|c|c|c|c|c|}
\hline $\mathbf{E}$ & $\mathbf{O}$ & $\mathbf{O}$ & $\mathbf{O}$ & $\mathbf{T}_{15}$ \\
\hline $\mathbf{O}$ & $\mathbf{E}$ & $\mathbf{O}$ & $\mathbf{O}$ & $\mathbf{T}_{25}$ \\
\hline $\mathbf{O}$ & $\mathbf{O}$ & $\mathbf{E}$ & $\mathbf{T}_{34}$ & $\mathbf{T}_{35}$ \\
\hline $\mathbf{O}$ & $\mathbf{O}$ & $\mathbf{O}$ & $\mathbf{E}$ & $\mathbf{O}$ \\
\hline $\mathbf{O}$ & $\mathbf{O}$ & $\mathbf{O}$ & $\mathbf{O}$ & $\mathbf{E}$ \\
\hline
\end{tabular}

where $\mathbf{T}_{15} \in \mathrm{M}_{t \times l_{4}}(D), \mathbf{T}_{i 5} \in \mathrm{M}_{l_{i-1} \times l_{4}}(D),(i=2,3)$ and $\mathbf{T}_{34} \in \mathrm{M}_{l_{2} \times l_{3}}(D)$ are matrices over $D$. The matrix of transformations $\mathbf{U}$ has the following form:

\begin{tabular}{|c|c|c|c|c|}
\hline $\mathbf{U}_{11}$ & $\mathbf{O}$ & $\mathbf{O}$ & $\mathbf{O}$ & $\mathbf{O}$ \\
\hline $\mathbf{O}$ & $\mathbf{U}_{22}$ & $\mathbf{O}$ & $\mathbf{O}$ & $\mathbf{O}$ \\
\hline $\mathbf{O}$ & $\mathbf{O}$ & $\mathbf{U}_{33}$ & $\mathbf{O}$ & $\mathbf{O}$ \\
\hline $\mathbf{O}$ & $\mathbf{O}$ & $\mathbf{O}$ & $\mathbf{U}_{44}$ & $\mathbf{O}$ \\
\hline
\end{tabular}




\section{\begin{tabular}{l|l|l|l|l|}
$\mathbf{O}$ & $\mathbf{O}$ & $\mathbf{O}$ & $\mathbf{O}$ & $\mathbf{U}_{55}$ \\
\hline
\end{tabular}}

where $\mathbf{U}_{11}$ is an invertible matrix with entries in $O$, and $\mathbf{U}_{i i}(i=2,3,4,5)$ are invertible matrices with entries in $D$. Reducing the matrix $\mathbf{T}$ by the matrix $\mathbf{U}$ is leads to the matrix problem given by a matrix $\mathbf{T}_{1}$ partitioned into 3 horizontal strips and 2 vertical strips:

\begin{tabular}{|l|l|}
\hline $\mathbf{O}$ & $\mathbf{A}_{1}$ \\
\hline $\mathbf{O}$ & $\mathbf{A}_{2}$ \\
\hline $\mathbf{A}_{4}$ & $\mathbf{A}_{3}$ \\
\hline
\end{tabular}

and the following admissible transformations:

(a) left $O$-elementary transformations with rows of the first horizontal strip of $\mathbf{T}_{1}$;

(b) left $D$-elementary transformations of rows of the second horizontal strip and third horizontal strip of $\mathbf{T}_{1}$;

(c) right $D$-elementary transformations of columns inside each vertical strip of $\mathbf{T}_{1}$.

Then one can reduce the second horizontal strip to the form:

\begin{tabular}{|cccccc|cccccc|}
\hline 0 & 0 & 0 & 0 & 0 & 0 & 0 & 0 & 0 & 0 & $\mathrm{E}$ & 0 \\
0 & 0 & 0 & 0 & 0 & 0 & 0 & 0 & $\mathrm{E}$ & 0 & 0 & 0 \\
0 & 0 & 0 & 0 & 0 & 0 & $\mathrm{E}$ & 0 & 0 & 0 & 0 & 0 \\
0 & 0 & 0 & 0 & 0 & 0 & 0 & 0 & 0 & 0 & 0 & 0 \\
\hline
\end{tabular}

and the third horizontal strip to the form:

\begin{tabular}{|cccccc|cccccc|}
\hline $\mathrm{E}$ & 0 & 0 & 0 & 0 & 0 & 0 & 0 & $\mathrm{E}$ & 0 & 0 & 0 \\
0 & $\mathrm{E}$ & 0 & 0 & 0 & 0 & 0 & 0 & 0 & $\mathrm{E}$ & 0 & 0 \\
0 & 0 & $\mathrm{E}$ & 0 & 0 & 0 & 0 & 0 & 0 & 0 & 0 & 0 \\
0 & 0 & 0 & $\mathrm{E}$ & 0 & 0 & 0 & 0 & 0 & 0 & 0 & 0 \\
0 & 0 & 0 & 0 & 0 & 0 & $\mathrm{E}$ & 0 & 0 & 0 & 0 & 0 \\
0 & 0 & 0 & 0 & 0 & 0 & 0 & $\mathrm{E}$ & 0 & 0 & 0 & 0 \\
0 & 0 & 0 & 0 & 0 & 0 & 0 & 0 & 0 & 0 & 0 & 0 \\
\hline
\end{tabular}

This process leads to the matrix problem given by a matrix $\mathbf{B}$ partitioned into 6 vertical strips

$$
\begin{array}{|l|l|l|l|l|l|}
\hline \mathbf{B}_{1} & \mathbf{B}_{2} & \mathbf{B}_{3} & \mathbf{B}_{4} & \mathbf{B}_{5} & \mathbf{B}_{6} \\
\hline
\end{array}
$$

and the following admissible transformations: 
(a) left $O$-elementary transformations with rows of the first horizontal strip of B;

(b) right $D$-elementary transformations with columns inside of each vertical strip $\mathbf{B}_{i}(i=1,2, . ., 6)$;

(c) right $D$-elementary transformations with columns of vertical strips $\mathbf{B}_{i}$ which are in one-to-one relation with the following poset $\mathrm{S}$ :

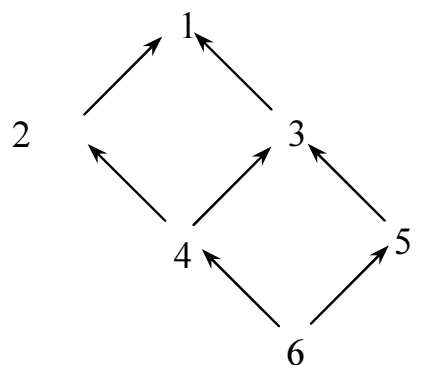

i.e., if $\alpha_{i} \leq \alpha_{j}$ in the poset $S$ then any column of the block $\mathbf{B}_{i}$ can be added to any column of the $\mathbf{B}_{j}$.

It is easy to see the blocks $\mathbf{B}_{2}, \mathbf{B}_{3}, \mathbf{B}_{4}$ form the matrix problem II, considered in [4]. Therefore ring $A$ is of unbounded representation type.

\section{Remark 4.}

A mixed matrix problem which forms matrices $\mathbf{B}_{i}(i=1, \ldots, 6)$ is defined by two posets: $P(X)$ is an infinite chain, and $P(Y)=\mathrm{S}$. Therefore the pair of posets $\{P(X)$, $P(Y)\}$ contains a critical pair of sets $\{(6),(1,2)\}$. By Theorem 4 this matrix problem is of unbounded representation type.

Lemma 11. Ring A corresponding to the diagram

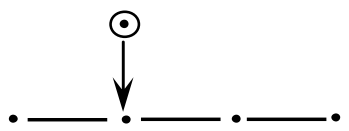

with arbitrary directions of arrows is a ring of unbounded representation type.

\section{Conclusions}

This paper proves the necessity in Theorem 1 for the ring $A(\mathrm{~S}, O)$, when all discrete valuation rings corresponding to minimal elements of the poset $\mathrm{S}$ are the same. In this case the necessity follows from Lemmas 5-11 and Propositions 2 and 3 .

\section{References}

[1] Gubareni N., Finitely presented modules over right hereditary SPSD-rings, Scientific Research of the Institute of Mathematics and Computer Science 2010, 2(9), 49-57. 
[2] Kirichenko V.V., Mogileva V.V., Pirus E.M., Khibina M.A., Semiperfect rings and piecewise domains, In: Algebraic Researches, Institute of Mathematics NAS Ukraine, 1995, 33-65 (in Russian).

[3] Dokuchaev M., Gubareni N., Rings connected with finite posets, Scientific Research of the Institute of Mathematics and Computer Science 2010, 2(9), 25-36.

[4] Gubareni N., Structure of finitely generated modules over right hereditary SPSD-rings, Scientific Research of the Institute of Mathematics and Computer Science 2012, 3(12), 45-56.

[5] Hazewinkel M., Gubareni N., Kirichenko V.V., Algebras, rings and modules. Vol. 1, Mathematics and Its Applications, v. 575, Kluwer Academic Publisher, Dordrecht-Boston-London 2004.

[6] Hazewinkel M., Gubareni N., Kirichenko V.V. Algebras, Rings and Modules. Vol. 2. Springer, 2007.

[7] Gubareni N.M., Kirichenko V.V., Revitskaya U.S., Semiperfect semidistributive semihereditary rings of modular restricted type, Proc. Gomel State Univ., Problems in Algebra 1999, 1(15), 29-47 (in Russian).

[8] Gubareni N.M., Right hereditary rings of bounded representation type, Preprint-148 Inst. Electrodynamics Akad. Nauk Ukrain. SSR, Kiev 1977, 48 p (in Russian).

[9] Zavadskij A.G., Revitskaya U.S., A matrix problem over a discrete valuation ring, Mat. Sb. 1999, 6(190), 59-82 (in Russian); English transl.: Sb. Math. 1999, 6, 835-858.

[10] Gabriel P., Indecomposable representations I, Manuscripta Math. 1972, 6, 71-103.

[11] Dlab V., Ringel C.M., On algebras of finite representation type, J. Algebra 1975, 33, 306-394.

[12] Kleiner M.M., Partially ordered sets of finite type, Zap. Nauchn. Sem. LOMI 1972, 28, 32-41 (in Russian); English transl.: J. Soviet Math. 1975, 3, 607-615. 